\title{
On a More Accurate Half-Discrete Mulholland-Type Inequality Involving One Multiple Upper Limit Function
}

\author{
Xianyong Huang $\mathbb{D}$ and Bicheng Yang \\ Department of Mathematics, Guangdong University of Education, Guangzhou, Guangdong 51003, China \\ Correspondence should be addressed to Xianyong Huang; huangxianyong2020@163.com
}

Received 14 June 2021; Accepted 11 November 2021; Published 7 December 2021

Academic Editor: Alexander Meskhi

Copyright (c) 2021 Xianyong Huang and Bicheng Yang. This is an open access article distributed under the Creative Commons Attribution License, which permits unrestricted use, distribution, and reproduction in any medium, provided the original work is properly cited.

By the use of the weight functions, the symmetry property, and Hermite-Hadamard's inequality, a more accurate half-discrete Mulholland-type inequality involving one multiple upper limit function is given. The equivalent conditions of the best possible constant factor related to multiparameters are studied. Furthermore, the equivalent forms, several inequalities for the particular parameters, and the operator expressions are provided.

\section{Introduction}

Suppose that $p>1,(1 / p)+(1 / q)=1, a_{m}, b_{n} \geq 0,0<\sum_{m=1}^{\infty} a_{m}^{p}$ $<\infty$,and $0<\sum_{n=1}^{\infty} b_{n}^{q}<\infty$. The following well-known HardyHilbert's inequality was provided (cf. [1], Theorem 315):

$$
\sum_{m=1}^{\infty} \sum_{n=1}^{\infty} \frac{a_{m} b_{n}}{m+n}<\frac{\pi}{\sin (\pi / p)}\left(\sum_{m=1}^{\infty} a_{m}^{p}\right)^{1 / p}\left(\sum_{n=1}^{\infty} b_{n}^{q}\right)^{1 / q}
$$

where the constant factor $\pi / \sin (\pi / p)$ is the best possible. Replacing $1 /(m+n)$ and $1 /(m+n-1)$ in (1), we have a more accurate form of (1) (cf. [1], Theorem 323). With regard to the similar assumptions, the following Mulholland's inequality was obtained (cf. [1], Theorem 343):

$$
\sum_{m=2}^{\infty} \sum_{n=2}^{\infty} \frac{a_{m} b_{n}}{\ln m n}<\frac{\pi}{\sin (\pi / p)}\left(\sum_{m=2}^{\infty} m^{p-1} a_{m}^{p}\right)^{1 / p}\left(\sum_{n=2}^{\infty} n^{q-1} b_{n}^{q}\right)^{1 / q} .
$$

In 2006, by using Euler-Maclaurin's summation formula and the technique of real analysis, Krnić and Pečarić [2] obtained an extension of (1) as follows:

$$
\sum_{m=1}^{\infty} \sum_{n=1}^{\infty} \frac{a_{m} b_{n}}{(m+n)^{\lambda}}<B\left(\lambda_{1}, \lambda_{2}\right)\left[\sum_{m=1}^{\infty} m^{p\left(1-\lambda_{1}\right)-1} a_{m}^{p}\right]^{1 / p}\left[\sum_{n=1}^{\infty} n^{q\left(1-\lambda_{2}\right)-1} b_{n}^{q}\right]^{1 / q},
$$

where $\lambda_{i} \in(0,2](i=1,2), \lambda_{1}+\lambda_{2}=\lambda \in(0,4]$, the constant factor $B\left(\lambda_{1}, \lambda_{2}\right)$ in (3) is the best possible, and

$$
B(u, v)=\int_{0}^{\infty} \frac{t^{u-1}}{(1+t)^{u+v}} d t(u, v>0)
$$

is the beta function. For $\lambda=1, \lambda_{1}=1 / q, \lambda_{2}=1 / p$ in (3), it reduces to (1); for $p=q=2$ and $\lambda_{1}=\lambda_{2}=\lambda / 2$ in (3), it reduces to Yang's inequality in [3].

Using Abel's partial sum formula and (2), in 2019, Adiyasuren et al. [4] provided a discrete Hardy-Hilbert's inequality with the kernel as (3) involving two partial sums. We find that inequalities (1)-(3) played an important role in analysis and its applications (cf. [5-14]).

In 2016, Hong and Wen [15] gave an equivalent condition of the best possible constant factor related to a few parameters in the general form of (1). In the same way, the other similar works of (1) and the integral analogues were given in [16-19].

In 1934, a half-discrete Hilbert-type inequality was published in Hardy et al. [1] (Theorem 351) as follows: if $K(t)(t>0)$ is decreasing, $p>1,(1 / p)+(1 / q)=1,0<\phi(s)=$ 
$\int_{0}^{\infty} K(t) t^{s-1} d t<\infty$, and $a_{n} \geq 0$, satisfying $0<\sum_{n=1}^{\infty} a_{n}^{p}<\infty$, then, we have

$$
\int_{0}^{\infty} x^{p-2}\left(\sum_{n=1}^{\infty} K(n x) a_{n}\right)^{p} d x<\phi^{p}\left(\frac{1}{q}\right) \sum_{n=1}^{\infty} a_{n}^{p}
$$

Some new extensions of (5) with their applications were provided by [20-24].

In this article, following the way of $[4,15]$, by the use of the weight functions, the symmetry property, and Hermite-Hadamard's inequality, a more accurate half-discrete Mulholland-type inequality with the kernel as $1 /\left[x+\ln ^{\alpha}(n-\xi)\right]^{\lambda}$ involving one multiple upper limit function is obtained. The equivalent conditions of the best possible constant factor related to multiparameters are given. Furthermore, the equivalent forms, several new inequalities for the particular parameters, and the operator expressions are considered. The lemmas and theorems of this paper provide an extensive account of this type of inequalities.

\section{Some Lemmas}

In what follows, we suppose that $p>1,(1 / p)+(1 / q)=1$, $N:=\{1,2, \cdots\}, \xi \in[0,1 / 2], \lambda>0, \lambda_{1} \in(0, \lambda), \lambda_{2} \in,(0, \lambda+m)$ $\cap(0,1 / \alpha], \widehat{\lambda}_{1}:=\left(\left(\lambda-\lambda_{2}\right) / p\right)+\left(\lambda_{1} / q\right), \widehat{\lambda}_{2}:=\left(\left(\lambda-\lambda_{1}\right) / q\right)+($ $\left.\lambda_{2} / p\right)$. We also assume that $f(x):=F_{0}(x)$ is a nonnegative Lebesgue integrable function in any interval $(0, b)(b>0)$. Define a multiple upper limit function as follows: $F_{i}(x):=\int_{0}^{x}$ $F_{i-1}(t) d t(x \geq 0)$, satisfying

$$
F_{i}(0)=0, F_{i}(x)=o\left(e^{t x}\right) \quad(t>0, x \longrightarrow \infty ; i=1, \cdots, m),
$$

and for any $F_{m}(x), a_{n} \geq 0$, it follows that

$$
\begin{aligned}
& 0<\int_{0}^{\infty} x^{-p\left(m-1+\lambda \wedge_{1}\right)-1} F_{m}^{p}(x)<\infty, \\
& 0<\sum_{n=2}^{\infty}[\ln (n-\xi)]^{q\left(1-\alpha \lambda \wedge_{2}\right)-1}(n-\xi)^{q-1} a_{n}^{q}<\infty .
\end{aligned}
$$

Lemma 1. Assuming that $s \in(0, \infty), s_{2} \in(0, s) \cap(0,1 / \alpha]$, $k_{s}\left(s_{2}\right):=B\left(s_{2}, s-s_{2}\right)$, define the following weight function:

$\omega\left(s_{2}, x\right):=\alpha x^{s-s_{2}} \sum_{n=2}^{\infty} \frac{[\ln (n-\xi)]^{\alpha s_{2}-1}}{\left[x+\ln ^{\alpha}(n-\xi)\right]^{s}(n-\xi)}\left(x \in R_{+}=(0, \infty)\right)$

The following inequality is valid:

$$
\omega\left(s_{2}, x\right)<k_{s}\left(s_{2}\right)\left(x \in R_{+}\right) .
$$

Proof. For fixed $x \in R_{+}$, the function $[\ln (t-\xi)]^{\alpha s_{2}-1}$, $\left[x+\ln ^{\alpha}(t-\xi)\right]^{s}(t-\xi)$ is strictly decreasing and strictly convex in the interval $(3 / 2, \infty)$. In fact, for $\alpha \in(0,1], s_{2} \in(0,1 / \alpha]$, $\xi \in[0,1 / 2], t \in(3 / 2, \infty)$, it follows that

$$
\begin{aligned}
& \frac{\partial}{\partial t} \frac{[\ln (t-\xi)]^{\alpha s_{2}-1}}{\left[x+\ln ^{\alpha}(t-\xi)\right]^{s}(t-\xi)}=-\frac{\left(1-\alpha s_{2}\right)[\ln (t-\xi)]^{\alpha s_{2}-2}}{\left[x+\ln ^{\alpha}(t-\xi)\right]^{s}(t-\xi)^{2}} \\
& -\frac{s \alpha[\ln (t-\xi)]^{\alpha s_{2}+\alpha-2}}{\left[x+\ln ^{\alpha}(t-\xi)\right]^{s+1}(t-\xi)^{2}} \\
& -\frac{[\ln (t-\xi)]^{\alpha s_{2}-1}}{\left[x+\ln ^{\alpha}(t-\xi)\right]^{s}(t-\xi)^{2}}<0, \\
& \frac{\partial^{2}}{\partial t^{2}} \frac{[\ln (t-\xi)]^{\alpha s_{2}-1}}{\left[x+\ln ^{\alpha}(t-\xi)\right]^{s}(t-\xi)}=\frac{\left(1-\alpha s_{2}\right)\left(2-\alpha s_{2}\right)[\ln (t-\xi)]^{\alpha s_{2}-3}}{\left[x+\ln ^{\alpha}(t-\xi)\right]^{s}(t-\xi)^{3}} \\
& +\frac{s \alpha\left(3-2 \alpha s_{2}-\varepsilon\right)[\ln (t-\xi)]^{\alpha s_{2}+\alpha-3}}{\left[x+\ln ^{\alpha}(t-\xi)\right]^{s+1}(t-\xi)^{3}} \\
& +\frac{3\left(1-\alpha s_{2}\right)[\ln (t-\xi)]^{\alpha s_{2}-2}}{\left[x+\ln ^{\alpha}(t-\xi)\right]^{s}(t-\xi)^{3}} \\
& +\frac{s \alpha^{2}(s+1)[\ln (t-\xi)]^{\alpha s_{2}+2 \alpha-3}}{\left[x+\ln ^{\alpha}(t-\xi)\right]^{s+2}(t-\xi)^{3}} \\
& +\frac{3 s \alpha[\ln (t-\xi)]^{\alpha s_{2}+\alpha-2}}{\left[x+\ln ^{\alpha}(t-\xi)\right]^{s+1}(t-\xi)^{3}} \\
& +\frac{2[\ln (t-\xi)]^{\alpha s_{2}-1}}{\left[x+\ln ^{\alpha}(t-\xi)\right]^{s}(t-\xi)^{3}}>0 \text {. }
\end{aligned}
$$

Applying Hermite-Hadamard's inequality (cf. [25]), we have

$$
\sum_{n=2}^{\infty} \frac{[\ln (n-\xi)]^{\alpha s_{2}-1}}{\left[x+\ln ^{\alpha}(n-\xi)\right]^{s}(n-\xi)}<\int_{3 / 2}^{\infty} \frac{[\ln (t-\xi)]^{\alpha s_{2}-1}}{\left[x+\ln ^{\alpha}(t-\xi)\right]^{s}(t-\xi)} d t
$$

Setting $\quad v=\ln ^{\alpha}(t-\xi) / x\left((1 /(t-\xi)) d t=(1 / \alpha) x^{1 / \alpha} v^{(1 / \alpha)-1}\right.$ $d v)$, it follows that

$$
\begin{aligned}
\int_{3 / 2}^{\infty} & \frac{[\ln (t-\xi)]^{\alpha s_{2}-1}}{\left[x+\ln ^{\alpha}(t-\xi)\right]^{s}(t-\xi)} d t \\
\quad= & \frac{1}{\alpha x^{s}} \int_{\ln ^{\alpha}((3 / 2)-\xi) / x}^{\infty} \frac{(x v)^{1 / \alpha\left(\alpha s_{2}-1\right)}}{(1+v)^{s}} x^{1 / \alpha} v^{1 / \alpha-1} d v \\
\quad \leq & \frac{1}{\alpha x^{s-s_{2}}} \int_{0}^{\infty} \frac{v^{s_{2}-1} d v}{(1+v)^{s}}=\frac{1}{\alpha x^{s-s_{2}}} k_{s}\left(s_{2}\right) .
\end{aligned}
$$

By (8) and (11), we have the following inequality:

$$
\omega\left(s_{2}, x\right)<\alpha x^{s-s_{2}} \frac{1}{\alpha x^{s-s_{2}}} k_{s}\left(s_{2}\right)=k_{s}\left(s_{2}\right),
$$

namely, (9) follows.

The lemma is prove.

Lemma 2. For $s_{1} \in(0, s)$ and $k_{s}\left(s_{1}\right)=B s_{1},\left(s-s_{1}\right)$, if

$$
0<\int_{0}^{\infty} x^{p\left[1-\left(\left(\left(s-s_{2}\right) / p\right)+\left(s_{1} / q\right)\right)\right]-1} f^{p}(x) d x<\infty
$$


$0<\sum_{n=2}^{\infty}\{\ln (n-\xi)]^{q\left[1-\alpha\left(\left(s_{2} / p\right)+\left(\left(s-s_{1}\right) / q\right)\right)\right]-1}(n-\xi)^{q-1} a_{n}^{q}<\infty$,

then we have the following more accurate half-discrete Mulholland-type inequality:

$$
\begin{aligned}
I_{0}:= & \int_{0}^{\infty} \sum_{n=2}^{\infty} \frac{a_{n}}{\left[x+\ln ^{\alpha}(n-\xi)\right]^{s}} f(x) d x \\
< & \left(\frac{1}{\alpha} k_{s}\left(s_{2}\right)\right)^{1 / p}\left(k_{s}\left(s_{1}\right)\right)^{1 / q} \\
& \times\left\{\int_{0}^{\infty} x^{p\left[1-\left(\left(\left(s-s_{2}\right) / p\right)+\left(s_{1} / q\right)\right)\right]-1} f^{p}(x) d x\right\}^{1 / p} \\
& \cdot\left\{\sum_{n=2}^{\infty}[\ln (n-\xi)]^{q\left[1-\alpha\left(\left(s_{2} / p\right)+\left(\left(s-s_{1}\right) / q\right)\right)\right]-1}(n-\xi)^{q-1} a_{n}^{q}\right\}^{1 / q} .
\end{aligned}
$$

Proof. Setting $v=x / \ln ^{\alpha}(n-\xi)$, we obtain the expression of the following another weight function:

$$
\begin{aligned}
\omega\left(s_{1}, n\right) & :=[\ln (n-\xi)]^{\alpha\left(s-s_{1}\right)} \int_{0}^{\infty} \frac{x^{s_{1}-1} d x}{\left[x+\ln ^{\alpha}(n-\xi)\right]^{s}} \\
& =\int_{0}^{\infty} \frac{v^{s_{1}-1} d v}{(v+1)^{s}}=k_{s}\left(s_{1}\right)(n \in N \backslash\{1\}) .
\end{aligned}
$$

Using Hölder's inequality (cf. [25]), it follows that

$$
\begin{aligned}
I_{0}= & \int_{0}^{\infty} \sum_{n=2}^{\infty} \frac{1}{\left[x+\ln ^{\alpha}(n-\xi)\right]^{s}}\left[\frac{x^{\left(1-s_{1}\right) / q}(n-\xi)^{-1 / p}}{[\ln (n-\xi)]^{\left(1-\alpha s_{2}\right) / p}} f(x)\right] \\
& \cdot\left[\frac{[\ln (n-\xi)]^{\left(1-\alpha s_{2}\right) / p}}{x^{\left(1-s_{1}\right) / q}(n-\xi)^{-1 / p}} a_{n}\right] d x \\
\leq & \left\{\int_{0}^{\infty} \sum_{n=2}^{\infty} \frac{(n-\xi)^{-1}}{\left[x+\ln ^{\alpha}(n-\xi)\right]^{s}} \frac{x^{\left(1-s_{1}\right)(p-1)}}{[\ln (n-\xi)]^{1-\alpha s_{2}}} f^{p}(x) d x\right\}^{1 / p} \\
& \times\left\{\sum_{n=2}^{\infty} \int_{0}^{\infty} \frac{1}{\left[x+\ln ^{\alpha}(n-\xi)\right]^{s}} \frac{[\ln (n-\xi)]^{\left(1-\alpha s_{2}\right)(q-1)} a_{n}^{q}}{x^{1-s_{1}}(n-\xi)^{1-q}} d x\right\}^{1 / q} \\
=\{ & \left.\frac{1}{\alpha} \int_{0}^{\infty} \omega\left(s_{2}, x\right) x^{p\left[1-\left(\left(s-s_{2}\right) / p+s_{1} / q\right)\right]-1} f^{p}(x) d x\right\}^{1 / p} \\
& \times\left\{\sum_{n=2}^{\infty} \omega\left(s_{1}, n\right)[\ln (n-\xi)]^{q\left[1-\alpha\left(\left(s-s_{1}\right) / q+s_{2} / p\right)\right]-1}(n-\xi)^{q-1} a_{n}^{q}\right\}^{1 / q} .
\end{aligned}
$$

We show that the above inequality does not keep the form of equality. Otherwise (cf. [25]), there exist constants
$A$ and $B$, such that they are not both zero and

$$
\begin{aligned}
& A \frac{x^{\left(1-s_{1}\right)(p-1)}(n-\xi)^{-1}}{[\ln (n-\xi)]^{1-\alpha s_{2}}} f^{p}(x) \\
& \quad=B \frac{[\ln (n-\xi)]^{\left(1-\alpha s_{2}\right)(q-1)} a_{n}^{q}}{x^{1-s_{1}}(n-\xi)^{1-q}} \text { a.e.in } R_{+} \times N \backslash\{1\} . .
\end{aligned}
$$

Suppose that $A \neq 0$. There exists a $n \in N \backslash\{1\}$, such that

$$
\begin{aligned}
x^{p\left[1-\alpha\left(\left(\left(s-s_{2}\right) / p\right)+\left(s_{1} / q\right)\right)\right]-1} f^{p}(x)= & \frac{B}{A}[\ln (n-\xi)]^{q\left(1-\alpha s_{2}\right)} \\
& \cdot(n-\xi)^{q} a_{n}^{q} \frac{1}{x^{1+\left(s-s_{1}-s_{2}\right)}} \text { a.e.in } R_{+},
\end{aligned}
$$

which contradicts the fact that

$$
0<\int_{0}^{\infty} x^{p\left[1-\left(\left(\left(\left(s-s_{2}\right) / p\right)\right)+\left(s_{1} / q\right)\right)\right]-1} f^{p}(x) d x<\infty,
$$

based on $\int_{0}^{\infty}\left(1 / x^{1+\left(s-s_{1}-s_{2}\right)}\right) d x=\infty$. Then, by (9) and (17), we have (16).

The lemma is proven.

Remark 1. Replacing $f(x)$ by $F_{m}(x)$ and setting $s=\lambda+m \in$ $(m, \infty), s_{1}=\lambda_{1}+m \in(m, s)$, and $s_{2}=\lambda_{2} \in(0, s) \cap(0,1 / \alpha]$ in (16), we find $\lambda \in(0, \infty), \lambda_{1} \in(0, \lambda)$, and $\lambda_{2} \in(0, \lambda+m) \cap(0$, $1 / \alpha]$ and obtain the following more accurate half-discrete Mulholland-type inequality with new parameters:

$$
\begin{aligned}
& \int_{0}^{\infty} \sum_{n=2}^{\infty} \frac{a_{n}}{\left[x+\ln ^{\alpha}(n-\xi)\right]^{\lambda+m}} F_{m}(x) d x \\
&<\left(\frac{1}{\alpha} k_{\lambda+m}\left(\lambda_{2}\right)\right)^{1 / p}\left(k_{\lambda+m}\left(\lambda_{1}+m\right)\right)^{1 / q} \\
& \quad \times\left[\int_{0}^{\infty} x^{-p\left(m-1+\lambda \wedge_{1}\right)-1} F_{m}^{p}(x) d x\right]^{1 / p} \\
& \quad \times\left\{\sum_{n=2}^{\infty}[\ln (n-\xi)]^{q\left(1-\alpha \lambda \wedge_{2}\right)-1}(n-\xi)^{q-1} a_{n}^{q}\right\}^{1 / q} .
\end{aligned}
$$

Lemma 3. For $t>0$, the following expression is valid:

$$
\int_{0}^{\infty} e^{-t x} f(x) d x=t^{m} \int_{0}^{\infty} e^{-t x} F_{m}(x) d x
$$

Proof. For $m=0$ since $F_{0}(x)=f(x),(23)$ is naturally valid; for $m \in N$, integration by parts, since $F_{i}(0)=0, F_{i}(x)=o\left(e^{t x}\right)$ $(t>0, i=1, \cdots m ; x \longrightarrow \infty)$, we find

$$
\int_{0}^{\infty} e^{-t x} F_{i-1}(x) d x=\int_{0}^{\infty} e^{-t x} d F_{i}(x)=\left.e^{-t x} F_{i}(x)\right|_{0} ^{\infty}-\int_{0}^{\infty} F_{i}(x) d e^{-t x}=\lim _{x \longrightarrow \infty} e^{-t x} F_{i}(x)+t \int_{0}^{\infty} e^{-t x} F_{i}(x) d x=t \int_{0}^{\infty} e^{-t x} F_{i}(x) d x
$$


By substitution of $i=1, \cdots, m$ in the above expression, we have (23).

The lemma is proven.

\section{Main Results}

Theorem 1. The following more accurate half-discrete Mulholland-type inequality involving one multiple upper limit function is valid:

$$
\begin{aligned}
I:= & \int_{0}^{\infty} \sum_{n=2}^{\infty} \frac{a_{n}}{\left[x+\ln ^{\alpha}(n-\xi)\right]^{\lambda}} f(x) d x \\
< & \frac{\Gamma(\lambda+m)}{\Gamma(\lambda)}\left(\frac{1}{\alpha} k_{\lambda+m}\left(\hat{\lambda}_{2}\right)\right)^{1 / p}\left(k_{\lambda+m}\left(\hat{\lambda}_{1}+m\right)\right)^{1 / q} \\
& \times\left[\int_{0}^{\infty} x^{-p\left(m-1+\overleftarrow{\lambda}_{1}\right)-1} F_{m}^{p}(x) d x\right]^{1 / p} \\
& \cdot\left\{\sum_{n=2}^{\infty}[\ln (n-\xi)]^{q\left(1-\alpha \overleftarrow{\lambda}_{2}\right)-1}(n-\xi)^{q-1} a_{n}^{q}\right\}^{1 / q} .
\end{aligned}
$$

In particular, for $\lambda_{1}+\lambda_{2}=\lambda\left(\lambda_{1} \in(0, \lambda), \lambda_{2} \in(0, \lambda) \cap(0\right.$, $1 / \alpha]$ ), we have

$0<\int_{0}^{\infty} x^{-p\left(m-1+\lambda_{1}\right)-1} F_{m}^{p}(x) d x<\infty, \quad 0<\sum_{n=2}^{\infty}[\ln (n-\xi)]^{q\left(1-\alpha \lambda_{2}\right)-1}(n-\xi)^{q-1} a_{n}^{q}<\infty$,

and the following inequality:

$$
\begin{aligned}
I= & \int_{0}^{\infty} \sum_{n=2}^{\infty} \frac{a_{n}}{\left[x+\ln ^{\alpha}(n-\xi)\right]^{\lambda}} f(x) d x \\
< & \frac{\Gamma(\lambda+m)}{\alpha^{1 / p} \Gamma(\lambda)} B\left(\lambda_{1}+m, \lambda_{2}\right) \\
& \times\left[\int_{0}^{\infty} x^{-p\left(m-1+\lambda_{1}\right)-1} F_{m}^{p}(x) d x\right]^{1 / p} \\
& \cdot\left\{\sum_{n=2}^{\infty}[\ln (n-\xi)]^{q\left(1-\alpha \lambda_{2}\right)-1}(n-\xi)^{q-1} a_{n}^{q}\right\}^{1 / q} .
\end{aligned}
$$

Proof. In view of the following expression:

$$
\frac{1}{\left[x+\ln ^{\alpha}(n-\xi)\right]^{\lambda}}=\frac{1}{\Gamma(\lambda)} \int_{0}^{\infty} t^{\lambda-1} e^{-\left[x+\ln ^{\alpha}(n-\xi)\right] t} d t
$$

by the Lebesgue term by the term integration theorem (cf. [26]) and (23), we obtain

$$
\begin{aligned}
I & =\frac{1}{\Gamma(\lambda)} \int_{0}^{\infty} \sum_{n=2}^{\infty} a_{n} f(x) \int_{0}^{\infty} t^{\lambda-1} e^{-\left[x+\ln ^{\alpha}(n-\xi)\right] t} d t d x \\
& =\frac{1}{\Gamma(\lambda)} \int_{0}^{\infty} t^{\lambda-1}\left(\int_{0}^{\infty} e^{-x t} f(x) d x\right) \sum_{n=2}^{\infty} e^{-t \ln ^{\alpha}(n-\xi)} a_{n} d t \\
& =\frac{1}{\Gamma(\lambda)} \int_{0}^{\infty} t^{\lambda-1}\left(t^{m} \int_{0}^{\infty} e^{-x t} F_{m}(x) d x\right) \sum_{n=2}^{\infty} e^{-t \ln ^{\alpha}(n-\xi)} a_{n} d t \\
& =\frac{1}{\Gamma(\lambda)} \int_{0}^{\infty} \sum_{n=2}^{\infty} a_{n} F_{m}(x)\left\{\int_{0}^{\infty} t^{(\lambda+m)-1} e^{-\left[x+\ln ^{\alpha}(n-\xi)\right] t} d t\right\} d x \\
& =\frac{\Gamma(\lambda+m)}{\Gamma(\lambda)} \int_{0}^{\infty} \sum_{n=1}^{\infty} \frac{a_{n}}{\left[x+\ln ^{\alpha}(n-\xi)\right]^{\lambda+m}} F_{m}(x) d x .
\end{aligned}
$$

Then, by (22), we have (25).

The theorem is proven.

Theorem 2. If $\lambda_{1}+\lambda_{2}=\lambda\left(\lambda_{1} \in(0, \lambda), \lambda_{2} \in(0, \lambda) \cap(0,1 / \alpha]\right)$, then the constant factor

$$
\frac{\Gamma(\lambda+m)}{\Gamma(\lambda)}\left(\frac{1}{\alpha} k_{\lambda+m}\left(\lambda_{2}\right)\right)^{1 / p}\left(k_{\lambda+m}\left(\lambda_{1}+m\right)\right)^{1 / q}
$$

is the best possible. On the other hand, if we add $\lambda_{2}<\lambda$, $\lambda-\lambda_{1} \leq 1 / \alpha$ and the same constant factor in (25) is the best possible, then we have $\lambda_{1}+\lambda_{2}=\lambda$.

Proof. If $\lambda_{1}+\lambda_{2}=\lambda$, then (25) reduces to (27). For any $0<$ $\varepsilon<p \lambda_{1}$, we set

$$
\begin{aligned}
\tilde{f}(x) & :=\left\{\begin{array}{ll}
0, & 0<x<1, \\
x^{\lambda_{1}-\varepsilon / p-1}, & x \geq 1,
\end{array} \tilde{a}_{n}\right. \\
& :=[\ln (n-\xi)]^{\alpha\left(\lambda_{2}-\varepsilon / q\right)-1}(n-\xi)^{-q}(n \in N \backslash\{1\}) .
\end{aligned}
$$

We have $\tilde{F}_{0}(x)=\tilde{f}(x)$ and

$$
\begin{aligned}
\tilde{F}_{i}(x) & :=\int_{0}^{x} \tilde{F}_{i-1}(t) d t \\
& \leq\left\{\begin{array}{ll}
0, & 0<x<1, \\
\frac{1}{\prod_{j=0}^{i-1}\left(\lambda_{1}+j-\varepsilon / p\right)} x^{\lambda_{1}+i-1-\varepsilon / p}, & x \geq 1,
\end{array} \quad(i \in\{1, \cdots, m\}),\right.
\end{aligned}
$$

satisfying $\tilde{F}_{i}(0)=0, \tilde{F}_{i}(x)=o\left(e^{t x}\right)(t>0, x \longrightarrow \infty ; i=1, \cdots, m)$. If there exists a positive constant $M\left(\leq\left(\Gamma(\lambda+m) / \alpha^{1 / p} \Gamma\right.\right.$ $\left.(\lambda)) k_{\lambda+m}\left(\lambda_{1}+m\right)\right)$, such that (27) is valid when we replace $\left(\Gamma(\lambda+m) / \alpha^{1 / p} \Gamma(\lambda)\right) k_{\lambda+m}\left(\lambda_{1}+m\right)$ by $M$, then in particular, it follows that 


$$
\begin{aligned}
\tilde{I}:= & \int_{0}^{\infty} \sum_{n=2}^{\infty} \frac{\tilde{a}_{n} \tilde{f}(x) d x}{\left[x+\ln ^{\alpha}(n-\xi)\right]^{\lambda}} \\
< & M\left[\int_{0}^{\infty} x^{-p\left(m-1+\lambda_{1}\right)-1} \tilde{F}_{m}^{p}(x) d x\right]^{1 / p} \\
& \cdot\left\{\sum_{n=2}^{\infty}[\ln (n-\xi)]^{q\left(1-\alpha \lambda_{2}\right)-1}(n-\xi)^{q-1} \tilde{a}_{n}^{q}\right\}^{1 / q} .
\end{aligned}
$$

For $m=0, \varepsilon \geq 0$, we define $\prod_{i=0}^{m-1}\left(\lambda_{1}+i-(\varepsilon / p)\right)=1$. By the decreasing property of the series, we find

$$
\begin{aligned}
\tilde{I}< & \frac{M}{\prod_{i=0}^{m-1}\left(\lambda_{1}+i-\varepsilon / p\right)}\left[\int_{1}^{\infty} x^{-p\left(m-1+\lambda_{1}\right)-1} x^{p\left(\lambda_{1}+m-1\right)-\varepsilon} d x\right]^{1 / p} \\
& \times\left\{\sum_{n=2}^{\infty}[\ln (n-\xi)]^{q\left(1-\alpha \lambda_{2}\right)-1}(n-\xi)^{q-1}[\ln (n-\xi)]^{q \alpha \lambda_{2}-\alpha \varepsilon-q}(n-\xi)^{-q}\right\}^{1 / q} \\
= & \frac{M}{\prod_{i=0}^{m-1}\left(\lambda_{1}+i-\varepsilon / p\right)}\left(\int_{1}^{\infty} x^{-\varepsilon-1} d x\right)^{1 / p} \\
& \times\left\{[\ln (2-\xi)]^{-\alpha \varepsilon-1}(2-\xi)^{-1}+\sum_{n=3}^{\infty}[\ln (n-\xi)]^{-\alpha \varepsilon-1}(n-\xi)^{-1}\right\}^{1 / q} \\
\leq & \frac{M}{\prod_{i=0}^{m-1}\left(\lambda_{1}+i-\varepsilon / p\right)}\left(\int_{1}^{\infty} x^{-\varepsilon-1} d x\right)^{1 / p} \\
& \times\left\{[\ln (2-\xi)]^{-\alpha \varepsilon-1}(2-\xi)^{-1}+\int_{2}^{\infty}[\ln (t-\xi)]^{-\alpha \varepsilon-1}(t-\xi)^{-1} d t\right\}^{1 / q} \\
= & \frac{M}{\varepsilon \prod_{i=0}^{m-1}\left(\lambda_{1}+i-\varepsilon / p\right)}\left\{\varepsilon[\ln (2-\xi)]^{-\alpha \varepsilon-1}(2-\xi)^{-1}+\frac{1}{\alpha}[\ln (2-\xi)]^{-\alpha \varepsilon}\right\}^{1 / q} .
\end{aligned}
$$

By (17), setting $s=\lambda$ and $s_{1}=\tilde{\lambda}_{1}:=\lambda_{1}-(\varepsilon / p) \in(0, \lambda)$ $\left(0<\tilde{\lambda}_{2}:=\lambda_{2}+(\varepsilon / p)<\lambda\right)$, it follows that

$$
\begin{aligned}
\tilde{I}= & \left.\sum_{n=2}^{\infty}\left\{[\ln (n-\xi)]^{\alpha\left(\lambda_{2}+\varepsilon \varepsilon p\right)} \int_{1}^{\infty} \frac{x^{\left(\lambda_{1}-\varepsilon / p\right)-1}}{\left[x+\ln ^{\alpha}(n-\xi)\right]^{\lambda}} d x\right\} \ln (n-\xi)\right]^{-\alpha \varepsilon-1}(n-\xi)^{-1} \\
= & \sum_{n=2}^{\infty} \omega\left(\tilde{\lambda}_{1}, n\right)[\ln (n-\xi)]^{-\alpha \varepsilon-1}(n-\xi)^{-1} \\
& -\sum_{n=2}^{\infty}[\ln (n-\xi)]^{\alpha\left(\tilde{\lambda}_{2}-\varepsilon\right)-1}(n-\xi)^{-1} \int_{0}^{1} \frac{x^{\tilde{\lambda}_{1}-1}}{\left[x+\ln ^{\alpha}(n-\xi)\right]^{\lambda}} d x \\
> & B\left(\tilde{\lambda}_{1}, \tilde{\lambda}_{2}\right) \sum_{n=2}^{\infty}[\ln (n-\xi)]^{-\alpha \varepsilon-1}(n-\xi)^{-1} \\
& -\sum_{n=2}^{\infty}[\ln (n-\xi)]^{\alpha\left(\tilde{\lambda}_{2}-\varepsilon\right)-1}(n-\xi)^{-1} \int_{0}^{1} \frac{x^{\tilde{\lambda}_{1}-1} d x}{\ln (n-\xi)]^{\alpha \lambda}} \\
\geq & B\left(\tilde{\lambda}_{1}, \tilde{\lambda}_{2}\right) \int_{2}^{\infty}[\ln (y-\xi)]^{-\alpha \varepsilon-1}(y-\xi)^{-1} d y \\
& -\frac{1}{\tilde{\lambda}_{1}} \sum_{n=2}^{\infty}[\ln (n-\xi)]^{-\alpha\left(\tilde{\lambda}_{1}+\varepsilon\right)-1}(n-\xi)^{-1} \\
= & \frac{1}{\varepsilon}\left\{\frac{1}{\alpha} B\left(\lambda_{1}-\frac{\varepsilon}{p}, \lambda_{2}+\frac{\varepsilon}{p}\right)[\ln (2-\xi)]^{-\alpha \varepsilon}-\varepsilon O(1)\right\} .
\end{aligned}
$$

In virtue of the above results, we have

$$
\begin{aligned}
& \frac{1}{\alpha} B(\left.\lambda_{1}-\frac{\varepsilon}{p}, \lambda_{2}+\frac{\varepsilon}{p}\right)[\ln (2-\xi)]^{-\alpha \varepsilon}-\varepsilon O(1) \\
&<\varepsilon \tilde{I}<\frac{M}{\prod_{i=0}^{m-1}\left(\lambda_{1}+i-(\varepsilon / p)\right)} \\
& \quad \times\left\{\varepsilon[\ln (2-\xi)]^{-\alpha \varepsilon-1}(2-\xi)^{-1}+\frac{1}{\alpha}[\ln (2-\xi)]^{-\alpha \varepsilon}\right]^{1 / q}
\end{aligned}
$$

For $\varepsilon \longrightarrow 0^{+}$, by the continuity of the beta function, we have

$$
\begin{aligned}
\frac{\Gamma(\lambda+m)}{\alpha^{1 / p} \Gamma(\lambda)} B\left(\lambda_{1}+m, \lambda_{2}\right) & =\frac{\Gamma\left(\lambda_{1}+m\right) \Gamma\left(\lambda_{2}\right)}{\alpha^{1 / p} \Gamma(\lambda)} \\
& =\frac{1}{\alpha^{1 / p}} B\left(\lambda_{1}, \lambda_{2}\right) \prod_{i=0}^{m-1}\left(\lambda_{1}+i\right) \\
& \leq M .
\end{aligned}
$$

Hence, $\quad M=\left(\Gamma(\lambda+m) / \alpha^{1 / p} \Gamma(\lambda)\right) B\left(\lambda_{1}+m, \lambda_{2}\right)$ is the best possible constant factor in (27).

On the other hand, for $\widehat{\lambda}_{1}=\left(\left(\lambda-\lambda_{2}\right) / p\right)+\left(\lambda_{1} / q\right), \widehat{\lambda}_{2}=$ $\left(\left(\lambda-\lambda_{1}\right) / q\right)+\left(\lambda_{2} / p\right)$, in view of $0<\lambda_{2}<\lambda, \lambda-\lambda_{1} \leq 1 / \alpha$, we find $\hat{\lambda}_{1}+\widehat{\lambda}_{2}=\left(\left(\lambda-\lambda_{2}\right) / p\right)+\left(\lambda_{1} / q\right)+\left(\left(\lambda-\lambda_{1}\right) / q\right)+\left(\lambda_{2} / p\right)$ $=\lambda, 0<\widehat{\lambda}_{1}, \widehat{\lambda}_{2}<(\lambda / p)+(\lambda / q)=\lambda, \widehat{\lambda}_{2} \leq((1 / \alpha) / q)+((1 / \alpha) /$ $p)=1 / \alpha$, and

$$
\frac{\Gamma(\lambda+m)}{\alpha^{1 / p} \Gamma(\lambda)} B\left(\widehat{\lambda}_{1}+m, \widehat{\lambda}_{2}\right) \in R_{+} \cdot \cdot
$$

By substitution of $\widehat{\lambda}_{i}=\lambda_{i}(i=1,2)$ in (27), we find

$$
\begin{aligned}
I= & \int_{0}^{\infty} \sum_{n=2}^{\infty} \frac{a_{n}}{\left.<x+\ln ^{\alpha}(n-\xi)\right]^{\lambda}} f(x) d x \\
< & \frac{\Gamma(\lambda+m)}{\alpha^{1 / p} \Gamma(\lambda)} k_{\lambda+m}\left(\widehat{\lambda}_{1}+m\right) \\
& \times\left[\int_{0}^{\infty} x^{-p\left(m-1+\lambda \wedge_{1}\right)-1} F_{m}^{p}(x) d x\right]^{1 / p} \\
& \cdot\left\{\sum_{n=2}^{\infty}[\ln (n-\xi)]^{q\left(1-\alpha \lambda \Lambda_{2}\right)-1}(n-\xi)^{-1} a_{n}^{q}\right\}^{1 / q} .
\end{aligned}
$$


By means of Hölder's inequality (cf. [25]), it follows that

$$
\begin{aligned}
k_{\lambda+m}\left(\hat{\lambda}_{1}+m\right)= & k_{\lambda+m}\left(\frac{\lambda-\lambda_{2}}{p}+\frac{\lambda_{1}}{q}+m\right) \\
= & \int_{0}^{\infty} \frac{1}{(1+u)^{\lambda+m}} u^{\left(\left(\lambda-\lambda_{2}+m\right) / p\right)+\left(\left(\lambda_{1}+m\right) / q\right)-1} d u \\
= & \int_{0}^{\infty} \frac{1}{(1+u)^{\lambda+m}}\left(u^{\left(\lambda-\lambda_{2}+m-1\right) / p}\right)\left(u^{\left(\lambda_{1}+m-1\right) / q}\right) d u \\
\leq & {\left[\int_{0}^{\infty} \frac{1}{(1+u)^{\lambda+m}} u^{\lambda-\lambda_{2}+m-1} d u\right]^{1 / p} } \\
& \cdot\left[\int_{0}^{\infty} \frac{1}{(1+u)^{\lambda+m}} u^{\lambda_{1}+m-1} d u\right]^{1 / q} \\
= & {\left[\int_{0}^{\infty} \frac{1}{(1+v)^{\lambda+m}} v^{\lambda_{2}-1} d v\right]^{1 / p} } \\
& \cdot\left[\int_{0}^{\infty} \frac{1}{(1+u)^{\lambda+m}} u^{\left(\lambda_{1}+m\right)-1} d u\right]^{1 / q} \\
= & \left(k_{\lambda+m}\left(\lambda_{2}\right)\right)^{1 / p}\left(k_{\lambda+m}\left(\lambda_{1}+m\right)\right)^{1 / q} .
\end{aligned}
$$

Since $\quad\left(\Gamma(\lambda+m) / \alpha^{1 / p} \Gamma(\lambda)\right)\left(k_{\lambda+m}\left(\lambda_{2}\right)\right)^{1 / p}\left(k_{\lambda+m} \quad\left(\lambda_{1}+\right.\right.$ $m))^{1 / q}$ is the best possible constant factor in (25), by (39), we have the following inequality:

$$
\begin{aligned}
& \frac{\Gamma(\lambda+m)}{\alpha^{1 / p} \Gamma(\lambda)}\left(k_{\lambda+m}\left(\lambda_{2}\right)\right)^{1 / p}\left(k_{\lambda+m}\left(\lambda_{1}+m\right)\right)^{1 / q} \\
& \quad \leq \frac{\Gamma(\lambda+m)}{\alpha^{1 \cdot p} \Gamma(\lambda)} k_{\lambda+m}\left(\hat{\lambda}_{1}+m\right)\left(\in R_{+}\right),
\end{aligned}
$$

namely, $k_{\lambda+m}\left(\widehat{\lambda}_{1}+m\right) \geq\left(k_{\lambda+m}\left(\lambda_{2}\right)\right)^{1 / p}\left(k_{\lambda+m}\left(\lambda_{1}+m\right)\right)^{1 / q}$, and then, (40) keeps the form of equality.

We observe that (40) keeps the form of equality if and only if there exist constants $A$ and $B$ (cf. [25]), such that they are not both zero and $A u^{\lambda-\lambda_{2}}=B u^{\lambda_{1}}$ a.e. in $R_{+}$. Suppose that $A \neq 0$. We find $u^{\lambda-\lambda_{2}-\lambda_{1}}=(B / A)$ a.e. in $R_{+}$, namely, $\lambda-\lambda_{2}-$ $\lambda_{1}=0$. Hence, it follows that $\lambda_{1}+\lambda_{2}=\lambda$.

The theorem is proven.

\section{Equivalent Forms and Some Particular Inequalities}

Theorem 3. The following half-discrete more accurate Mulholland-type inequality involving a multiple upper limit function is equivalent to (25):

$$
\begin{aligned}
J:= & \left\{\sum_{n=2}^{\infty}[\ln (n-\xi)]^{p \alpha \lambda \wedge_{2}-1}(n-\xi)^{-1}\left[\int_{0}^{\infty} \frac{f(x)}{\left[x+\ln ^{\alpha}(n-\xi)\right]^{\lambda}} d x\right]^{p}\right\}^{1 / p} \\
< & \frac{\Gamma(\lambda+m)}{\alpha^{1 / p} \Gamma(\lambda)}\left(k_{\lambda+m}\left(\lambda_{2}\right)\right)^{1 / p}\left(k_{\lambda+m}\left(\lambda_{1}+m\right)\right)^{1 / q} \\
& \cdot\left[\int_{0}^{\infty} x^{-p\left(m-1+\lambda \wedge_{1}\right)-1} F_{m}^{p}(x) d x\right]^{1 / p} .
\end{aligned}
$$

In particular, for $\lambda_{1}+\lambda_{2}=\lambda\left(\lambda_{1} \in(0, \lambda), \lambda_{2} \in(0, \lambda) \cap(0\right.$, $1 / \alpha])$, we have the following Mulholland-type inequality equivalent to (27):

$$
\begin{gathered}
\left\{\sum_{n=2}^{\infty}[\ln (n-\xi)]^{p \alpha \lambda_{2}-1}(n-\xi)^{-1}\left[\int_{0}^{\infty} \frac{f(x)}{\left[x+\ln ^{\alpha}(n-\xi)\right]^{\lambda}} d x\right]^{p}\right\}^{1 / p} \\
<\frac{\Gamma(\lambda+m)}{\alpha^{1 / p} \Gamma(\lambda)} B\left(\lambda_{1}+m, \lambda_{2}\right)\left[\int_{0}^{\infty} x^{-p\left(m-1+\lambda_{1}\right)-1} F_{m}^{p}(x) d x\right]^{1 / p} .
\end{gathered}
$$

Proof. By assuming that (42) is valid, by Hölder's inequality (cf. [25]), it follows that

$$
\begin{aligned}
I= & \sum_{n=2}^{\infty}\left\{[\ln (n-\xi)]^{\alpha \lambda \wedge_{2}-1 / p}(n-\xi)^{-1 / p} \int_{0}^{\infty} \frac{f(x) d x}{\left[x+\ln ^{\alpha}(n-\xi)\right]^{\lambda}}\right\} \\
& \times\left\{[\ln (n-\xi)]^{-\alpha \lambda \wedge_{2}+1 / p}(n-\xi)^{1 / p} a_{n}\right\} \\
\leq & J\left\{\sum_{n=2}^{\infty}[\ln (n-\xi)]^{q\left(1-\alpha \lambda \wedge_{2}\right)-1}(n-\xi)^{-1} a_{n}^{q}\right\}^{1 / q} .
\end{aligned}
$$

Then, by (42), we have (25).

On the other hand, suppose that (25) is valid. We set

$$
a_{n}:=[\ln (n-\xi)]^{p \alpha \lambda_{2}-1}(n-\xi)^{-1}\left[\int_{0}^{\infty} \frac{f(x)}{\left[x+\ln ^{\alpha}(n-\xi)\right]^{\lambda}} d x\right]^{p-1}, \quad n \in N \backslash\{1\} .
$$

If $J=0$, then (42) is naturally valid; if $J=\infty$, then it is impossible that makes (42) valid, i.e., $J<\infty$. By assuming that $0<J<\infty$, by (25), it follows that

$$
\begin{aligned}
0< & \sum_{n=2}^{\infty}[\ln (n-\xi)]^{q\left(1-\alpha \lambda \wedge_{2}\right)-1}(n-\xi)^{-1} a_{n}^{q}=J^{p} \\
= & I<\frac{\Gamma(\lambda+m)}{\alpha^{1 / p} \Gamma(\lambda)}\left(k_{\lambda+m}\left(\lambda_{2}\right)\right)^{1 / p}\left(k_{\lambda+m}\left(\lambda_{1}+m\right)\right)^{1 / q} \\
& \cdot\left[\int_{0}^{\infty} x^{-p\left(m-1+\lambda \wedge_{1}\right)-1} F_{m}^{p}(x) d x\right]^{1 / p} J^{p-1}<\infty, \\
J= & \left\{\sum_{n=2}^{\infty}[\ln (n-\xi)]^{q\left(1-\lambda \wedge_{2}\right)-1}(n-\xi)^{-1} a_{n}^{q}\right\}^{1 / p} \\
< & \frac{\Gamma(\lambda+m)}{\alpha^{1 / p} \Gamma(\lambda)}\left(k_{\lambda+m}\left(\lambda_{2}\right)\right)^{1 / p}\left(k_{\lambda+m}\left(\lambda_{1}+m\right)\right)^{1 / q} \\
& \cdot\left[\int_{0}^{\infty} x^{-p\left(m-1+\lambda \wedge_{1}\right)-1} F_{m}^{p}(x) d x\right]^{1 / p},
\end{aligned}
$$

namely, (42) follows, which is equivalent to (25).

The theorem is proven.

Theorem 4. If $\lambda_{1}+\lambda_{2}=\lambda\left(\lambda_{1} \in(0, \lambda), \lambda_{2} \in(0, \lambda) \cap(0,1 / \alpha]\right)$, then the constant factor

$$
\frac{\Gamma(\lambda+m)}{\alpha^{1 / p} \Gamma(\lambda)}\left(k_{\lambda+m}\left(\lambda_{2}\right)\right)^{1 / p}\left(k_{\lambda+m}\left(\lambda_{1}+m\right)\right)^{1 / q}
$$


in (42) is the best possible. On the other hand, if we add $\lambda_{2}$ $<\lambda, \lambda-\lambda_{1} \leq 1 / \alpha$ and the same constant factor in (42) is the best possible, then we have $\lambda_{1}+\lambda_{2}=\lambda$.

Proof. If $\lambda_{1}+\lambda_{2}=\lambda$, then by Theorem 2, the constant factor

$$
\frac{\Gamma(\lambda+m)}{\alpha^{1 / p} \Gamma(\lambda)}\left(k_{\lambda+m}\left(\lambda_{2}\right)\right)^{1 / p}\left(k_{\lambda+m}\left(\lambda_{1}+m\right)\right)^{1 / q}
$$

in (25) is the best possible. By (44), the constant factor in (42) is still the best possible. Otherwise, we would reach a contradiction that the constant factor in (25) is not the best possible.

On the other hand, if the constant factor in (42) is the best possible, then by the equivalency of (42) and (25), in view of $J^{P}=I$ (see the proof of Theorem 3), we still can show that the constant factor in (25) is the best possible. By the assumptions and Theorem 2, it follows that $\lambda_{1}+\lambda_{2}=\lambda$.

The theorem is proven.

\section{Example 1.}

(i) For $\alpha=1$, (27) and (43) reduce to the following equivalent inequalities:

$$
\begin{aligned}
\int_{0}^{\infty} \sum_{n=2}^{\infty} \frac{a_{n}}{\ln ^{\lambda} e^{x}(n-\xi)} f(x) d x \\
<\times\left[\int_{0}^{\infty} x^{-p\left(m-1+\lambda_{1}\right)-1} F_{m}^{p}(x) d x\right]^{1 / p} \\
\quad \times\left\{\sum_{n=2}^{\infty}[\ln (n-\xi)]^{q\left(1-\lambda_{2}\right)-1}(n-\xi)^{q-1} a_{n}^{q}\right\}^{1 / q},
\end{aligned}
$$

$$
\begin{gathered}
\left\{\sum_{n=2}^{\infty}[\ln (n-\xi)]^{p \lambda_{2}-1}(n-\xi)^{-1}\left[\int_{0}^{\infty} \frac{f(x)}{\ln ^{\lambda} e^{x}(n-\xi)} d x\right]^{p}\right\}^{1 / p} \\
<B\left(\lambda_{1}, \lambda_{2}\right) \prod_{i=0}^{m-1}\left(\lambda_{1}+i\right)\left[\int_{0}^{\infty} x^{-p\left(m-1+\lambda_{1}\right)-1} F_{m}^{p}(x) d x\right]^{1 / p} .
\end{gathered}
$$

(ii) For $\xi=0$, (27) and (43) reduce to the following equivalent inequalities:

$$
\begin{aligned}
\int_{0}^{\infty} & \sum_{n=2}^{\infty} \frac{a_{n}}{\left(x+\ln ^{\alpha} n\right)^{\lambda}} f(x) d x<\frac{1}{\alpha^{1 / p}} B\left(\lambda_{1}, \lambda_{2}\right) \prod_{i=0}^{m-1}\left(\lambda_{1}+i\right) \\
\times & {\left[\int_{0}^{\infty} x^{-p\left(m-1+\lambda_{1}\right)-1} F_{m}^{p}(x) d x\right]^{1 / p} } \\
\cdot & {\left[\sum_{n=2}^{\infty}(\ln n)^{q\left(1-\alpha \lambda_{2}\right)-1} n^{q-1} a_{n}^{q}\right]^{1 / q}, }
\end{aligned}
$$

$$
\begin{aligned}
& \left\{\sum_{n=2}^{\infty} \frac{(\ln n)^{p \alpha \lambda_{2}-1}}{n}\left[\int_{0}^{\infty} \frac{f(x)}{\left(x+\ln ^{\alpha} n\right)^{\lambda}} d x\right]^{p}\right\}^{1 / p} \\
& \quad<\frac{1}{\alpha^{1 / p}} B\left(\lambda_{1}, \lambda_{2}\right) \prod_{i=0}^{m-1}\left(\lambda_{1}+i\right)\left[\int_{0}^{\infty} x^{-p\left(m-1+\lambda_{1}\right)-1} F_{m}^{p}(x) d x\right]^{1 / p} .
\end{aligned}
$$

Hence, (27) (resp. (43)) is a more accurate form of (51) (resp. (52)).

(iii) For $\xi=1 / 2$, (27) and (43) reduce to the following equivalent inequalities:

$$
\begin{aligned}
& \int_{0}^{\infty} \sum_{n=2}^{\infty} \frac{a_{n}}{\left[x+\ln ^{\alpha}(n-1 / 2)\right]^{\lambda}} f(x) d x<\frac{1}{\alpha^{1 / p}} B\left(\lambda_{1}, \lambda_{2}\right) \prod_{i=0}^{m-1}\left(\lambda_{1}+i\right) \\
& \times\left[\int_{0}^{\infty} x^{-p\left(m-1+\lambda_{1}\right)-1} F_{m}^{p}(x) d x\right]^{1 / p} \\
& \cdot\left\{\sum_{n=2}^{\infty}\left[\ln \left(n-\frac{1}{2}\right)\right]^{q\left(1-\alpha \lambda_{2}\right)-1}\left(n-\frac{1}{2}\right)^{q-1} a_{n}^{q}\right\}^{1 / q}, \\
&\left\{\sum_{n=2}^{\infty}\left[\ln \left(n-\frac{1}{2}\right)\right]^{p \alpha \lambda_{2}-1}\left(n-\frac{1}{2}\right)^{-1}\left[\int_{0}^{\infty} \frac{f(x)}{[x+\ln \alpha(n-1 / 2)]^{\lambda}} d x\right]^{p}\right\}^{1 / p} \\
& \quad<\frac{1}{\alpha^{1 / p}} B\left(\lambda_{1}, \lambda_{2}\right) \prod_{i=0}^{m-1}\left(\lambda_{1}+i\right)\left[\int_{0}^{\infty} x^{-p\left(m-1+\lambda_{1}\right)-1} F_{m}^{p}(x) d x\right]^{1 / p} .
\end{aligned}
$$

The constant factors in the above inequalities are still the best possible.

\section{Operator Expressions}

Setting $\phi_{m}(x):=x^{-p\left(m-1+\lambda \wedge_{1}\right)-1}, \psi(n):=[\ln (n-\xi)]^{q\left(1-\alpha \lambda \wedge_{2}\right)-1}$ $(n-\xi)^{q-1}$, from

$\psi^{1-p}(n)=[\ln (n-\xi)]^{p \alpha \lambda \wedge_{2}-1}(n-\xi)^{-1} \quad\left(x \in R_{+}, n \in N \backslash\{1\}\right)$,

we define the following normed linear spaces:

$$
\begin{aligned}
L_{p, \phi_{m}}\left(R_{+}\right) & :=\left\{f=f(x) ;\|f\|_{p, \phi_{m}}:=\left(\int_{0}^{\infty} \phi_{m}(x)|f(x)|^{p} d x\right)^{1 / p}<\infty\right\}, \\
l_{q, \psi} & :=\left\{a=\left\{a_{n}\right\}_{n=2}^{\infty} ;\|a\|_{q, \psi}:=\left(\sum_{n=2}^{\infty} \psi(n)\left|a_{n}\right|^{q}\right)^{1 / q}<\infty\right\}, \\
l_{p, \psi^{1-p}} & =\left\{c=\left\{c_{n}\right\}_{n=2}^{\infty} ;\|c\|_{p, \psi^{1-p}}:=\left(\sum_{n=2}^{\infty} \psi^{1-p}(n)\left|c_{n}\right|^{p}\right)^{1 / p}<\infty\right\} .
\end{aligned}
$$

For any $f=F_{0}(x) \in L_{p, \phi_{0}}\left(R_{+}\right)$, setting

$$
c=\left\{c_{n}\right\}_{n=2}^{\infty}: c_{n}:=\int_{0}^{\infty} \frac{f(x)}{\left[x+\ln ^{\alpha}(n-\xi)\right]^{\lambda}} d x,
$$


we can rewrite (42) as follows:

$$
\|c\|_{p, \psi^{1-p}}<\frac{\Gamma(\lambda+m)}{\alpha^{1 / p} \Gamma(\lambda)}\left(k_{\lambda+m}\left(\lambda_{2}\right)\right)^{1 / p}\left(k_{\lambda+m}\left(\lambda_{1}+m\right)\right)^{1 / q}\left\|F_{m}\right\|_{p, \phi_{m}}<\infty,
$$

namely, $c \in l_{p, \psi^{1-p}}$.

Definition 1. Define a half-discrete Mulholland-type operator $T: L_{p, \phi_{0}}\left(R_{+}\right) \longrightarrow l_{p, \psi^{1-p}}$ as follows: for any $f \in L_{p, \phi_{0}}\left(R_{+}\right)$, there exists a unique representation $c=T f \in l_{p, \psi^{1-p}}$, satisfying for any $n \in N \backslash\{1\} T f(n)=c_{n}$. Define the formal inner product of $T f$ and $a \in l_{q, \psi}$ and the norm of $T$ as follows:

$$
\begin{gathered}
(T f, a):=\sum_{n=2}^{\infty} a_{n} \int_{0}^{\infty} \frac{f(x)}{\left[x+\ln ^{\alpha}(n-\xi)\right]^{\lambda}} d x=I, \\
\|T\|:=\sup _{f(\neq 0) \in L_{p, \phi_{0}}\left(R_{+}\right)} \frac{\|T f\|_{p, \psi^{1-p}}}{\left\|F_{m}\right\|_{p, \phi_{m}}} .
\end{gathered}
$$

By Theorem 3 and Theorem 4, it follows that.

Theorem 5. If $f(\geq 0) \in L_{p, \phi_{0}}\left(R_{+}\right), a(\geq 0) \in l_{q, \psi},\|f\|_{p, \phi_{0}}>0$, $\|a\|_{q, \psi}>0$, then we have the following equivalent inequalities:

$$
(T f, a)<\frac{\Gamma(\lambda+m)}{\alpha^{1 / p} \Gamma(\lambda)}\left(k_{\lambda+m}\left(\lambda_{2}\right)\right)^{1 / p}\left(k_{\lambda+m}\left(\lambda_{1}+m\right)\right)^{1 / q}\left\|F_{m}\right\|_{p, \phi_{m}}\|a\|_{q, \psi^{\prime}},
$$

$$
\|T f\|_{p, \psi^{1-p}}<\frac{\Gamma(\lambda+m)}{\alpha^{1 / p} \Gamma(\lambda)}\left(k_{\lambda+m}\left(\lambda_{2}\right)\right)^{1 / p}\left(k_{\lambda+m}\left(\lambda_{1}+m\right)\right)^{1 / q}\left\|F_{m}\right\|_{p, \phi_{m}} .
$$

$w ?>$ Moreover, if $\lambda_{1}+\lambda_{2}=\lambda$, then the constant factor $\left(\Gamma(\lambda+m) / \alpha^{1 / p} \Gamma(\lambda)\right)\left(k_{\lambda+m}\left(\lambda_{2}\right)\right)^{1 / p}\left(k_{\lambda+m}\left(\lambda_{1}+m\right)\right)^{1 / q}$ in (59) and (60) is the best possible, namely,

$$
\|T\|=\frac{\Gamma(\lambda+m)}{\alpha^{1 / p} \Gamma(\lambda)} B\left(\lambda_{1}+m, \lambda_{2}\right)
$$

On the other hand, if we add $\lambda_{2}<\lambda, \lambda-\lambda_{1} \leq 1 / \alpha$ and the constant factor in (59) and (60) is the best possible, then we have $\lambda_{1}+\lambda_{2}=\lambda$.

\section{Data Availability}

The data used to support the findings of this study are included within the article.

\section{Conflicts of Interest}

The authors declare that they have no competing interest.

\section{Acknowledgments}

This work is supported by the National Natural Science Foundation of China (No. 12071491), the Construction Project of
Teaching Quality and Teaching Reform in Guangdong Undergraduate Colleges and Universities in 2018 (Speciality of Financial Mathematics), and the Characteristic Innovation Project of Guangdong Provincial Colleges and Universities in 2020 (No. 2020KTSCX088). We are grateful for this help.

\section{References}

[1] G. H. Hardy, J. E. Littlewood, and G. Polya, Inequalities, Cambridge University Press, Cambridge, USA, 2nd edition edition, 1934.

[2] M. Krnić and J. Pečarić, "Extension of Hilbert's inequality," Journal of mathematical analysis and applications, vol. 324, no. 1, pp. 150-160, 2006.

[3] B. C. Yang, "On a generalization of Hilbert double series theorem," Journal of Nanjing University Mathematical Biquarterly, vol. 18, no. 1, pp. 145-152, 2001.

[4] V. Adiyasuren, T. Batbold, and L. E. Azar, "A new discrete Hilbert-type inequality involving partial sums," Journal of Inequalities and Applications, vol. 2019, no. 1, 2019.

[5] I. Perić and P. Vuković, "Multiple Hilbert's type inequalities with a homogeneous kernel," Banach Journal of Mathematical Analysis, vol. 5, no. 2, pp. 33-43, 2011.

[6] Q. L. Huang, "A new extension of a Hardy-Hilbert-type inequality," Journal of Inequalities and Applications, vol. 2015, no. $1,2015$.

[7] B. He, "A multiple Hilbert-type discrete inequality with a new kernel and best possible constant factor," Journal of Mathematical Analysis and Applications, vol. 431, no. 2, pp. 889-902, 2015.

[8] Z. T. Xie, Z. Zeng, and Y. F. Sun, "A new Hilbert-type inequality with the homogeneous kernel of degree-2," Advances and Applications in Mathematical Sciences, vol. 12, no. 7, pp. 391-401, 2013.

[9] Z. Zheng, R. R. Gandhi, and Z. T. Xie, "A new Hilbert-type inequality with the homogeneous kernel of degree-2 and with the integral," Bulletin of Mathematical Sciences and Applications, vol. 7, no. 1, pp. 9-17, 2014.

[10] D. M. Xin, "A Hilbert-type integral inequality with the homogeneous kernel of zero degree," Mathematical Theory and Applications, vol. 30, no. 2, pp. 70-74, 2010.

[11] L. E. Azar, "The connection between Hilbert and Hardy inequalities," Journal of Inequalities and Applications, vol. 2013, no. 1, 2013.

[12] V. Adiyasuren, T. Batbold, and M. Krnić, "Hilbert-type inequalities involving differential operators, the best constants and applications," Mathematical Inequalities \& Applications, vol. 18, pp. 111-124, 2015.

[13] C. J. Zhao and W. S. Cheung, "On Hilbert's inequalities with alternating signs," Journal of Mathematical Inequalities, vol. 12, no. 1, pp. 191-200, 2018.

[14] M. H. You and X. Sun, "On a Hilbert-type inequality with the kernel involving extended Hardy operator," Journal of Mathematical Inequalities, vol. 15, no. 3, pp. 1239-1253, 2021.

[15] Y. Hong and Y. M. Wen, "A necessary and sufficient condition of that Hilbert type series inequality with homogeneous kernel has the best constant factor," Annals Mathematica, vol. 37A, no. 3, pp. 329-336, 2016.

[16] Y. Hong, "On the structure character of Hilbert's type integral inequality with homogeneous kernel and application," Journal of Jilin University (Science Edition), vol. 55, no. 2, pp. 189-194, 2017. 
[17] Y. Hong, Q. L. Huang, B. C. Yang, and J. Q. Liao, “The necessary and sufficient conditions for the existence of a kind of Hilbert-type multiple integral inequality with the nonhomogeneous kernel and its applications," Journal of Inequalities and Applications, vol. 2017, no. 1, 2017.

[18] D. M. Xin, B. C. Yang, and A. Z. Wang, "Equivalent property of a Hilbert-type integral inequality related to the beta function in the whole plane," Journal of Function Spaces, vol. 2018, Article ID 2691816, 8 pages, 2018.

[19] Y. Hong, B. He, and B. C. Yang, "Necessary and sufficient conditions for the validity of Hilbert type integral inequalities with a class of quasi-homogeneous kernels and its application in operator theory," Journal of Mathematics Inequalities, vol. 12, no. 3, pp. 777-788, 2018.

[20] B. C. Yang and M. Krnić, "A half-discrete Hilbert-type inequality with a general homogeneous kernel of degree 0 ," Journal of Mathematical Inequalities, vol. 6, no. 3, pp. 401417, 2012.

[21] B. C. Yang and L. Debnath, Half-Discrete Hilbert-Type Inequalities, World Scientific Publishing, Singapore, 2014.

[22] L. E. Azar, "Two new forms of half-discrete Hilbert inequality," Journal of the Egyptian Mathematical Society, vol. 22, no. 2, pp. 254-257, 2014.

[23] A.-O. K. Nizar, L. E. Azar, and A. H. A. Bataineh, "A sharper form of half-discrete Hilbert inequality related to Hardy inequality," Univerzitet u Nišu, vol. 32, no. 19, pp. 67336740, 2018.

[24] Q. Chen and B. Yang, "On two kinds of the reverse halfdiscrete Mulholland-type inequalities involving higher-order derivative function," Journal of Inequalities and Applications, vol. 2021, no. 1, 2021.

[25] J. C. Kuang, Applied Inequalities, Shangdong Science and Technology Press, Jinan, China, 2004.

[26] J. C. Kuang, Real and Functional Analysis (Continuation), vol. 2, Higher Education Press, Beijing, China, 2015. 